\title{
Feedback Chaotic Synchronization with Disturbances
}

\author{
Mingjun Wang, Wanbo Yu, Jing Zhao \\ School of Information Engineering, Dalian University, Dalian, China \\ Email:wmjhome@163.com
}

How to cite this paper: Wang, M.J., Yu, W.B. and Zhao, J. (2017) Feedback Chaotic Synchronization with Disturbances. International Journal of Modern Nonlinear Theory and Application, 6, 1-10.

https://doi.org/10.4236/ijmnta.2017.61001

Received: September 20, 2016

Accepted: January 8, 2017

Published: January 11, 2017

Copyright $\odot 2017$ by authors and Scientific Research Publishing Inc. This work is licensed under the Creative Commons Attribution International License (CC BY 4.0).

http://creativecommons.org/licenses/by/4.0/

\begin{abstract}
Based on Lyapunov stability theorem, a method is proposed for feedback synchronization with parameters perturbation and external disturbances. It is proved theoretically that if the perturbation and disturbances are bounded, the synchronization error can be ensured to approach to and stay within the pre-specified bound which can be arbitrarily small. Some typical chaotic systems with different types of nonlinearity, such as Lorenz system and the original Chua's circuit, are used for detailed description. The simulation results show the feasibility of the method.
\end{abstract}

\section{Keywords}

Lyapunov Stability Theorem, Feedback Synchronization, Parameters

Perturbation, External Disturbances, Robustness

\section{Introduction}

In 1990, Pecora and Carroll presented the conception of "chaotic synchronization" and introduced a method to synchronize two identical chaotic systems with different initial conditions [1] [2]. Since chaos control and synchronization have great potential applications in many areas such as information science, medicine, biology and engineering, they have received a great deal of attention. Numerous researches have been done theoretically and experimentally [3] [4] [5]. Muradi and Kapitaniak expanded Corroll and Pecora's work, presented a single unidirectional coupled synchronization scheme [6] [7]. Celka achieved chaos synchronization by using the time-delay feedback method [8]. Agiza et al. synchronized Rössler and Chen systems via active control method [9] and Impulsive control [10]. Guo et al. proposed a simple adaptive-feedback controller for chaos synchronization [11]. Agrawal et al. realized the synchronization of fractional order chaotic systems using active control method [12]. Norelys et al. 
presented the adaptive synchronization of fractional Lorenz systems using a reduced number of control signals and parameters [13]. Kajbaf et al. used sliding mode controller to obtain chaotic systems [14]. Wang et al. proposed a new feedback synchronization criterion based on the largest Lyapunov exponent [15]. However, most synchronization criterions were obtained under ideal circumstances. If parameters perturbation and external disturbance exist, this kind of criterions will take no effect. According to this practical problem, some solutions have been presented. For examples, Jiang et al. proposed a LMI criterion [16] for chaotic feedback synchronization. Although the simulations showed that it is robust to a random noise with zero mean, but no rigorous mathematical proof was provided and we can't determine if their method is effective for other kinds of noise. In Ref. [17], parameters perturbation was involved in their scheme. The theoretical proof and numerical simulations were given in their work, but external disturbance didn't receive attention, which made their method unilateral.

Above all, these methods are effective, but still lack generality or robustness. In this paper, we propose a practical synchronization scheme for chaotic synchronization with parameters perturbation and external disturbance. Rigorous mathematical proof is provided, and simulation results show the feasibility and robustness of our scheme.

\section{Theory and Method}

In the following scheme, a universal robust synchronization method is proposed. In the method, synchronization will be achieved with bounded parameter disturbances and noise.

Suppose a class of ideal chaotic systems as

$$
\dot{\boldsymbol{X}}=\boldsymbol{A} \boldsymbol{X}+f(\boldsymbol{X})
$$

where $\boldsymbol{A} \boldsymbol{X}$ is the linear part, $f(\boldsymbol{X})$ is the nonlinear part, then the system can be described as

$$
\dot{\boldsymbol{X}}=(\boldsymbol{A}+\Delta \boldsymbol{A}(t)) \boldsymbol{X}+f(\boldsymbol{X})+\Delta f(\boldsymbol{X}, t)+\boldsymbol{D}(t)
$$

where $\Delta \boldsymbol{A}(t)$ and $\Delta f(\boldsymbol{X}, t)$ are the parameters perturbation, $\boldsymbol{D}(t)$ is the external disturbance. Choose system (1) as the drive system, the relevant response system can be described as

$$
\dot{\boldsymbol{Y}}=\left(\boldsymbol{A}+\Delta \boldsymbol{A}^{\prime}(t)\right) \boldsymbol{Y}+f(\boldsymbol{Y})+\Delta f^{\prime}(\boldsymbol{Y}, t)-\boldsymbol{K}(\boldsymbol{Y}-\boldsymbol{X})+\boldsymbol{D}^{\prime}(t)
$$

where $\Delta \boldsymbol{A}^{\prime}(t), \Delta f^{\prime}(\boldsymbol{Y}, t)$ and $\boldsymbol{D}^{\prime}(t)$ are the relevant disturbances in the response system. We choose $\boldsymbol{K}=\operatorname{diag}\left(k_{1}, k_{2}, \cdots, k_{n}\right)$ ( $n$ is the dimension of the chaotic system). Let the error vector $\boldsymbol{E}=\boldsymbol{Y}-\boldsymbol{X}$, then the error is

$$
\begin{aligned}
\dot{\boldsymbol{E}}= & (\boldsymbol{A}-\boldsymbol{K}) \boldsymbol{E}+f(\boldsymbol{Y})-f(\boldsymbol{X})+\Delta \boldsymbol{A}^{\prime}(t) \boldsymbol{Y}-\Delta \boldsymbol{A}(t) \boldsymbol{X} \\
& +\Delta f^{\prime}(\boldsymbol{Y}, t)-\Delta f(\boldsymbol{X}, t)+\boldsymbol{D}^{\prime}(t)-\boldsymbol{D}(t)
\end{aligned}
$$

Set a pre-defined bound $\varepsilon$ for the synchronization error, suppose

$\boldsymbol{E}=\left[e_{1}, e_{2}, \cdots, e_{n}\right]^{\mathrm{T}}$, choose suitable $\boldsymbol{K}$ to ensure $\lim _{t \rightarrow \infty}\left\|e_{i}(t)\right\| \leq \varepsilon$ 
$(i=1,2, \cdots, n-1, n)$, then system (1) and system (2) achieve approximate synchronization, the precision is $\varepsilon$. When $\varepsilon$ is very small, we can consider system (1) and system (2) have been synchronized.

Choose the following Lyapunov function $V=\frac{1}{2} \sum_{i=1}^{n} e_{i}^{2}$, yield $\dot{V}=\sum_{i=1}^{n} e_{i} \dot{e}_{i}$.

According to Equation (3), the derivative of $e_{j}$ can be described as

$$
\dot{e}_{j}=\sum_{i=1}^{n} a_{j i} e_{i}+\sum_{i=1}^{n} h_{j i}(\boldsymbol{X}, \boldsymbol{Y}) e_{i}+g_{j}(\boldsymbol{X}, \boldsymbol{Y})+d_{j}-k_{j} e_{j}
$$

$a_{j i}$ is the element of matrix $\boldsymbol{A}, h_{j i}(\boldsymbol{X}, \boldsymbol{Y})$ and $g_{j}(\boldsymbol{X}, \boldsymbol{Y})$ is bounded, $d_{j}$ is bounded external disturbances, $k_{j}$ is feedback coefficients. When the errors go beyond $\varepsilon$, we have

$$
\begin{aligned}
& e_{j} \dot{e}_{j} \leq \frac{\left|g_{j}(\boldsymbol{X}, \boldsymbol{Y})+d_{j}\right|}{\varepsilon} e_{j}^{2}-k_{j} e_{j}^{2}+\sum_{i=1}^{n} \frac{\left|a_{j i}\right|}{2}\left(e_{i}^{2}+e_{j}^{2}\right)+\sum_{i=1}^{n} \frac{\left|h_{j i}(\boldsymbol{X}, \boldsymbol{Y})\right|}{2}\left(e_{i}^{2}+e_{j}^{2}\right) \\
\dot{V}= & \sum_{j=1}^{n} e_{j} \dot{e}_{j} \\
\leq & \sum_{j=1}^{n}\left(\frac{\left|g_{j}(\boldsymbol{X}, \boldsymbol{Y})+d_{j}\right|}{\varepsilon} e_{j}^{2}-k_{j} e_{j}^{2}+\sum_{i=1}^{n} \frac{\left|a_{j i}\right|}{2}\left(e_{i}^{2}+e_{j}^{2}\right)+\sum_{i=1}^{n} \frac{\left|h_{j i}(\boldsymbol{X}, \boldsymbol{Y})\right|}{2}\left(e_{i}^{2}+e_{j}^{2}\right)\right) \\
= & \sum_{j=1}^{n}\left(\frac{\left|g_{j}(\boldsymbol{X}, \boldsymbol{Y})+d_{j}\right|}{\varepsilon}-k_{j}+\sum_{i=1}^{n} \frac{\left|a_{j i}\right|}{2}+\sum_{i=1}^{n} \frac{\left|a_{i j}\right|}{2}+\sum_{i=1}^{n} \frac{\left|h_{j i}(\boldsymbol{X}, \boldsymbol{Y})\right|}{2}+\sum_{i=1}^{n} \frac{\left|h_{i j}(\boldsymbol{X}, \boldsymbol{Y})\right|}{2}\right) e_{j}^{2} .
\end{aligned}
$$

If

$$
k_{j}>\frac{\left|g_{j}(\boldsymbol{X}, \boldsymbol{Y})\right|+\left|d_{j}\right|}{\varepsilon}+\sum_{i=1}^{n} \frac{\left|a_{j i}\right|}{2}+\sum_{i=1}^{n} \frac{\left|a_{i j}\right|}{2}+\sum_{i=1}^{n} \frac{\left|h_{j i}(\boldsymbol{X}, \boldsymbol{Y})\right|}{2}+\sum_{i=1}^{n} \frac{\left|h_{j i}(\boldsymbol{X}, \boldsymbol{Y})\right|}{2}
$$

we can obtain

$$
\dot{V}=\sum_{i=1}^{n} e_{i} \dot{e}_{i}<0
$$

That is to say, when the error is not within the bound $\varepsilon$, it will exponentially converge to zero. Hence system (1) and system (2) will achieve approximate synchronization, the precision is $\varepsilon$ at least.

\section{Numerical Simulations}

Lorenz system and the original Chua's circuit have different types of nonlinearity. Next we will adopt the two systems for detailed description.

\subsection{Taking Lorenz System as Example}

Lorenz system [18] is described as

$$
\left\{\begin{array}{l}
\dot{x}=a(y-x) \\
\dot{y}=c x-y-x z \\
\dot{z}=x y-b z
\end{array}\right.
$$

In the paper choose $a=10, b=8 / 3, c=28$ so that system (9) exhibits a chaotic behavior [18]. The projections of Lorenz system's attractor are shown in 
Figure 1. Obviously we have $|x| \leq 20,|y| \leq 30,|z| \leq 50$.

Choose the following Lorenz system with parameters perturbation and external disturbances

$$
\left\{\begin{array}{l}
\dot{x}_{1}=\left(a+\xi_{a}\right)\left(x_{2}-x_{1}\right)+d_{1} \\
\dot{x}_{2}=\left(c+\xi_{c}\right) x_{1}-x_{2}-x_{1} x_{3}+d_{2} \\
\dot{x}_{3}=x_{1} x_{2}-\left(b+\xi_{b}\right) x_{3}+d_{3}
\end{array}\right.
$$

as drive system, then the relevant response system is

$$
\left\{\begin{array}{l}
\dot{y}_{1}=\left(a+\xi_{a}^{\prime}\right)\left(y_{2}-y_{1}\right)+d_{1}^{\prime}-k_{1}\left(y_{1}-x_{1}\right) \\
\dot{y}_{2}=\left(c+\xi_{c}^{\prime}\right) y_{1}-y_{2}-y_{1} y_{3}+d_{2}^{\prime}-k_{2}\left(y_{2}-x_{2}\right) \\
\dot{y}_{3}=y_{1} y_{2}-\left(b+\xi_{b}^{\prime}\right) y_{3}+d_{3}^{\prime}-k_{3}\left(y_{3}-x_{3}\right)
\end{array}\right.
$$

In system (10) and system (11), $\xi_{a}, \xi_{b}, \xi_{c}, \xi_{a}^{\prime}, \xi_{b}^{\prime}, \xi_{c}^{\prime}$ are parameters perturbation, $d_{1}, d_{2}, d_{3}, d_{1}^{\prime}, d_{2}^{\prime}, d_{3}^{\prime}$ are external disturbances, $k_{1}, k_{2}, k_{3}$ are feedback coefficients. Let

$$
\left\{\begin{array}{l}
e_{1}=y_{1}-x_{1} \\
e_{2}=y_{2}-x_{2} \\
e_{3}=y_{3}-x_{3}
\end{array}\right.
$$

Then $\dot{e}_{1}=\dot{y}_{1}-\dot{x}_{1}, \dot{e}_{2}=\dot{y}_{2}-\dot{x}_{2}, \quad \dot{e}_{3}=\dot{y}_{3}-\dot{x}_{3}$. The error system is

$$
\left\{\begin{array}{l}
\dot{e}_{1}=a\left(e_{2}-e_{1}\right)+\xi_{a}^{\prime}\left(y_{2}-y_{1}\right)-\xi_{a}\left(x_{2}-x_{1}\right)+d_{1}^{\prime}-d_{1}-k_{1} e_{1} \\
\dot{e}_{2}=c e_{1}-e_{2}-\left(y_{3} e_{1}+x_{1} e_{3}\right)+\xi_{c}^{\prime} y_{1}-\xi_{c} x_{1}+d_{2}^{\prime}-d_{1}-k_{2} e_{2} \\
\dot{e}_{3}=-b e_{3}+y_{2} e_{1}+x_{1} e_{2}-\left(\xi_{b}^{\prime} y_{3}-\xi_{b} x_{3}\right)+d_{3}^{\prime}-d_{3}-k_{3} e_{3}
\end{array}\right.
$$

Hence

$$
\left\{\begin{array}{l}
e_{1} \dot{e}_{1} \leq \frac{a}{2}\left(e_{1}^{2}+e_{2}^{2}\right)-a e_{1}^{2}+l_{1} e_{1}^{2}-k_{1} e_{1}^{2} \\
e_{2} \dot{e}_{2} \leq \frac{c}{2}\left(e_{1}^{2}+e_{2}^{2}\right)-e_{2}^{2}+\frac{\left|y_{3}\right|}{2}\left(e_{1}^{2}+e_{2}^{2}\right)+\frac{\left|x_{1}\right|}{2}\left(e_{2}^{2}+e_{3}^{2}\right)+l_{2} e_{2}^{2}-k_{2} e_{2}^{2} \\
e_{3} \dot{e}_{3} \leq-b e_{3}{ }^{2}+\frac{\left|y_{2}\right|}{2}\left(e_{2}^{2}+e_{3}^{2}\right)+\frac{\left|x_{1}\right|}{2}\left(e_{2}^{2}+e_{3}^{2}\right)+l_{3} e_{3}^{2}-k_{3} e_{3}^{2}
\end{array}\right.
$$

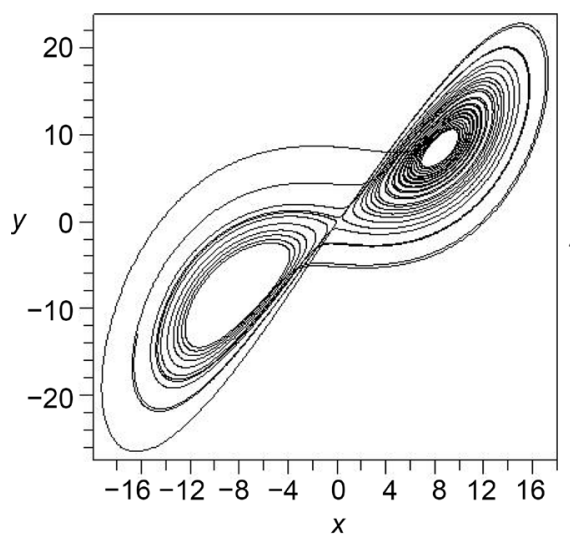

(a)

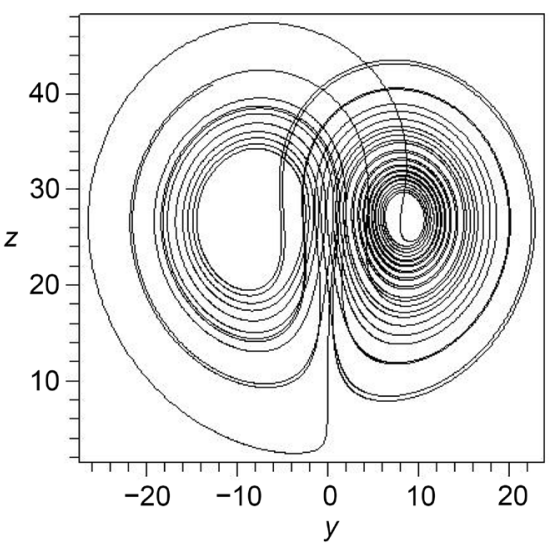

(b)

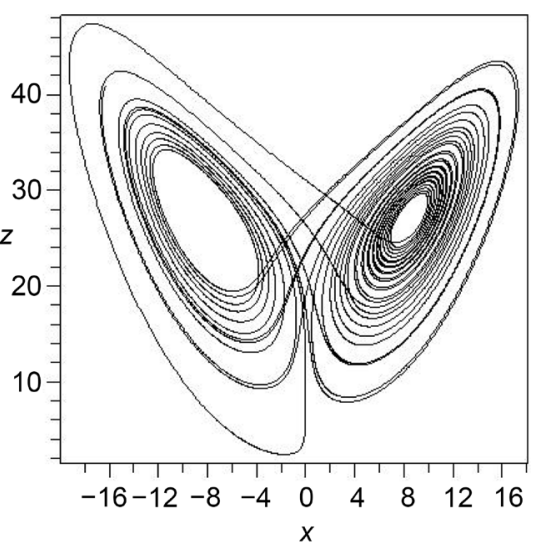

(c)

Figure 1. The projections of Lorenz system's attractor. 
where

$$
\left\{\begin{array}{l}
l_{1}=\frac{\left|\xi_{a}^{\prime}\right|\left(\left|y_{2}\right|+\left|y_{1}\right|\right)+\left|\xi_{a}\right|\left(\left|x_{2}\right|+\left|x_{1}\right|\right)+\left|d_{1}^{\prime}\right|+\left|d_{1}\right|}{\varepsilon} \\
l_{2}=\frac{\left|\xi_{c}^{\prime}\right|\left|y_{1}\right|+\left|\xi_{c}\right|\left|x_{1}\right|+\left|d_{2}^{\prime}\right|+\left|d_{1}\right|}{\varepsilon} \\
l_{3}=\frac{\left|\xi_{b}^{\prime}\right|\left|y_{3}\right|+\left|\xi_{b}\right|\left|x_{3}\right|+\left|d_{3}^{\prime}\right|+\left|d_{3}\right|}{\varepsilon}
\end{array}\right.
$$

Choose Lyapunov function

$$
V(t)=\frac{1}{2}\left(e_{1}^{2}+e_{2}^{2}+e_{3}^{2}\right)
$$

We have

$$
\dot{V}(t)=e_{1} \dot{e}_{1}+e_{2} \dot{e}_{2}+e_{3} \dot{e}_{3}
$$

Substitute Equation (14) into Equation (17), obtain

$$
\begin{aligned}
\dot{V}= & \left(l_{1}+\frac{c-a+\left|y_{3}\right|+\left|y_{2}\right|}{2}-k_{1}\right) e_{1}^{2}+\left(l_{2}-1+\frac{a+c+\left|y_{3}\right|}{2}+\left|x_{1}\right|-k_{2}\right) e_{2}^{2} \\
& +\left(l_{3}-b+\frac{\left|y_{2}\right|}{2}+\left|x_{1}\right|-k_{3}\right) e_{3}^{2} .
\end{aligned}
$$

If

$$
\left\{\begin{array}{l}
k_{1}>l_{1}+\frac{c-a+\left|y_{3}\right|+\left|y_{2}\right|}{2} \\
k_{2}>l_{2}-1+\frac{a+c+\left|y_{3}\right|}{2}+\left|x_{1}\right| \\
k_{3}>l_{3}-b+\frac{\left|y_{2}\right|}{2}+\left|x_{1}\right|
\end{array}\right.
$$

is satisfied, we will obtain $\dot{V}(t)<0$. According to Lyapunov stability theorem, the error system (13) will converge to zero when the error is not within the bound $\varepsilon$, i.e. system (10) and system (11) will achieve approximate synchronization, the precision is $\varepsilon$ at least.

When the parameters perturbation and external disturbances are small, we can consider the variables of system (10) and system (11) are bounded as shown in Figure 1. Suppose the upper bounds of these disturbances and perturbation are 0.5, choose $\varepsilon=0.1$, substitute Equation (15) into Equation (18), after calculating we obtain if

$$
\left\{\begin{array}{l}
k_{1}>559 \\
k_{2}>273 \\
k_{3}>543
\end{array}\right.
$$

is satisfied, Equation (18) will be always true.

In the simulation, suppose $\xi_{a}=0.5 \sin (2 t), \xi_{b}=0.5 \cos (t)$, $\xi_{c}=0.5 \cos (t+1), \quad \xi_{a}^{\prime}=0.5 \cos (3 t+2), \quad \xi_{b}^{\prime}=0.5 \sin (5 t), \quad \xi_{c}^{\prime}=0.5 \sin (2 t)$, $d_{1}, d_{2}, d_{3}, d_{1}^{\prime}, d_{2}^{\prime}, d_{3}^{\prime}$ are random from -0.5 to 0.5 . A time step of size 0.0001 (sec.) is employed and fourth-order Runge-Kutta method is used to solve Equation (10) 
and Equation (11). Let $k_{1}=560, k_{2}=280, k_{3}=550$, Figure 2 shows the history of $e_{1}(t), e_{2}(t), e_{3}(t)$ in the error system (13) within $0.1 \mathrm{sec}$. From Figure 2, we can see that $e_{1}(t), e_{2}(t), e_{3}(t)$ are steady near zero at last.

\subsection{Taking the Original Chua's Circuit as Example}

The original Chua's circuit [19] is described as

$$
\left\{\begin{array}{l}
\dot{x}=a(y-x-f(x)) \\
\dot{y}=x-y+z \\
\dot{z}=-b y
\end{array}\right.
$$

where $f(x)=d x+0.5(c-d)(|x+1|-|x-1|)$. In this paper choose $a=9.78$, $b=14.97, c=-1.31$ and $d=-0.75$ so that system (20) exhibits a chaotic behavior [19]. The projections of the original Chua's circuit's attractor are shown in Figure 3. Obviously we have $|x| \leq 4,|y| \leq 1,|z| \leq 5.5$.

Choose the following Chua's circuit with parameters perturbation and external disturbances

$$
\left\{\begin{array}{l}
\dot{x}_{1}=\left(a+\xi_{a}\right)\left(x_{2}-x_{1}-f\left(x_{1}\right)\right)+d_{1} \\
\dot{x}_{2}=x_{1}-x_{2}+x_{3}+d_{2} \\
\dot{x}_{3}=-\left(b+\xi_{b}\right) x_{2}+d_{3}
\end{array}\right.
$$

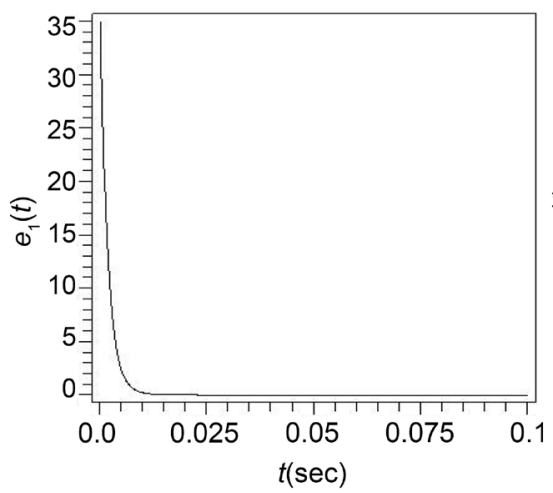

(a)

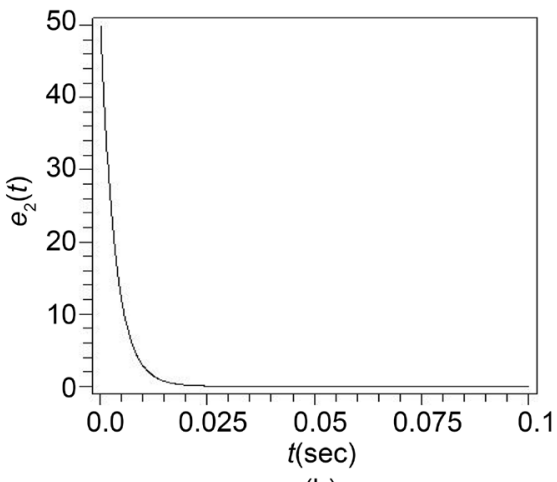

(b)

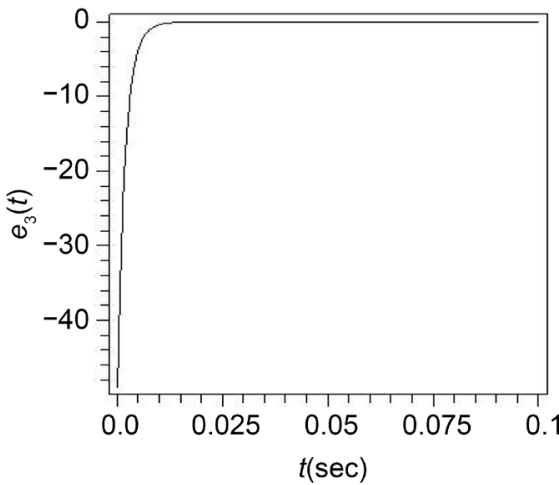

(c)

Figure 2. The history of the error (within $0.1 \mathrm{sec}$.).

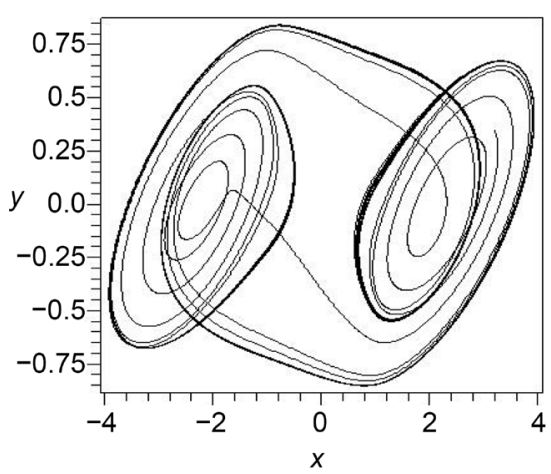

(a)

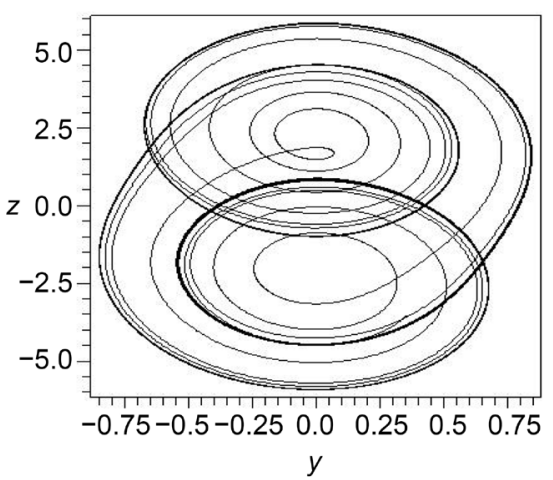

(b)

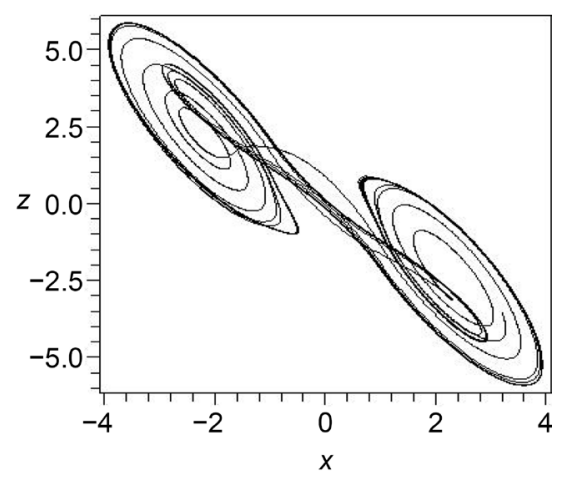

(c)

Figure 3. The projections of the original Chua's circuit's attractor. 
As drive system, where

$f\left(x_{1}\right)=\left(d+\xi_{d}\right) x_{1}+0.5\left(\left(c+\xi_{c}\right)-\left(d+\xi_{d}\right)\right)\left(\left|x_{1}+1\right|-\left|x_{1}-1\right|\right)$, then relevant response system is

$$
\left\{\begin{array}{l}
\dot{y}_{1}=\left(a+\xi_{a}^{\prime}\right)\left(y_{2}-y_{1}-f\left(y_{1}\right)\right)+d_{1}^{\prime}-k_{1}\left(y_{1}-x_{1}\right) \\
\dot{y}_{2}=y_{1}-y_{2}+y_{3}+d_{2}^{\prime}-k_{2}\left(y_{2}-x_{2}\right) \\
\dot{y}_{3}=-\left(b+\xi_{b}^{\prime}\right) y_{2}+d_{3}^{\prime}-k_{3}\left(y_{3}-x_{3}\right)
\end{array}\right.
$$

where $f\left(y_{1}\right)=\left(d+\xi_{d}^{\prime}\right) y_{1}+0.5\left(\left(c+\xi_{c}^{\prime}\right)-\left(d+\xi_{d}^{\prime}\right)\right)\left(\left|y_{1}+1\right|-\left|y_{1}-1\right|\right)$. In system (21) and system (22), $\xi_{a}, \xi_{b}, \xi_{c}, \xi_{d}, \xi_{a}^{\prime}, \xi_{b}^{\prime}, \xi_{c}^{\prime}, \xi_{d}^{\prime}$ are parameters perturbation,

$d_{1}, d_{2}, d_{3}, d_{1}^{\prime}, d_{2}^{\prime}, d_{3}^{\prime}$ are external disturbances, $k_{1}, k_{2}, k_{3}$ are feedback coefficients. Let

$$
\left\{\begin{array}{l}
e_{1}=y_{1}-x_{1} \\
e_{2}=y_{2}-x_{2} \\
e_{3}=y_{3}-x_{3}
\end{array}\right.
$$

Then $\dot{e}_{1}=\dot{y}_{1}-\dot{x}_{1}, \dot{e}_{2}=\dot{y}_{2}-\dot{x}_{2}, \dot{e}_{3}=\dot{y}_{3}-\dot{x}_{3}$. The error system is

$$
\left\{\begin{array}{l}
\dot{e}_{1}=a\left(e_{2}-e_{1}-\left(f\left(y_{1}\right)-f\left(x_{1}\right)\right)\right)+\xi_{a}^{\prime}\left(y_{2}-y_{1}-f\left(y_{1}\right)\right)-\xi_{a}\left(x_{2}-x_{1}-f\left(x_{1}\right)\right)+d_{1}^{\prime}-d_{1}-k_{1} e_{1} \\
\dot{e}_{2}=e_{1}-e_{2}+e_{3}+d_{2}^{\prime}-d_{1}-k_{2} e_{2} \\
\dot{e}_{3}=-b e_{2}-\left(\xi_{b}^{\prime} y_{2}-\xi_{b} x_{2}\right)+d_{3}^{\prime}-d_{3}-k_{3} e_{3}
\end{array}\right.
$$

when the parameters perturbation and external disturbances are small, we can consider the variables of system (21) and system (22) are bounded as shown in Figure 4 . Next we will substitute $|x| \leq 4,|y| \leq 1,|z| \leq 5.5$ directly to simplify the results, so we have

$$
\begin{aligned}
& \sup \left\{f\left(x_{1}\right)\right\} \leq 4\left(|d|+\left|\xi_{d}\right|\right)+|c|+\left|\xi_{c}\right|+|d|+\left|\xi_{d}\right|=5\left(|d|+\left|\xi_{d}\right|\right)+|c|+\left|\xi_{c}\right| \\
& \sup \left\{f\left(y_{1}\right)\right\} \leq 4\left(|d|+\left|\xi_{d}^{\prime}\right|\right)+|c|+\left|\xi_{c}^{\prime}\right|+|d|+\left|\xi_{d}^{\prime}\right|=5\left(|d|+\left|\xi_{d}^{\prime}\right|\right)+|c|+\left|\xi_{c}^{\prime}\right|
\end{aligned}
$$

Because

$$
\begin{aligned}
\left(\left|y_{1}+1\right|-\left|y_{1}-1\right|\right)-\left(\left|x_{1}+1\right|-\left|x_{1}-1\right|\right) & =\left(\left|y_{1}+1\right|-\left|x_{1}+1\right|\right)+\left(\left|x_{1}-1\right|-\left|y_{1}-1\right|\right) \\
& \leq\left|\left(y_{1}+1\right)-\left(x_{1}+1\right)\right|+\left|\left(x_{1}-1\right)-\left(y_{1}-1\right)\right| \\
& =2\left|e_{1}\right|,
\end{aligned}
$$

we have

$$
\begin{aligned}
\sup \left\{f\left(y_{1}\right)-f\left(x_{1}\right)\right\} & \leq|d|\left|e_{1}\right|+4\left|\xi_{d}^{\prime}\right|+4\left|\xi_{d}\right|+|(c-d)|\left|e_{1}\right|+\left(\left|\xi_{d}^{\prime}\right|+\left|\xi_{d}\right|+\left|\xi_{c}^{\prime}\right|+\left|\xi_{c}\right|\right) \\
& =|d|\left|e_{1}\right|+5\left|\xi_{d}^{\prime}\right|+5\left|\xi_{d}\right|+|c-d|\left|e_{1}\right|+\left|\xi_{c}^{\prime}\right|+\left|\xi_{c}\right| .
\end{aligned}
$$

Hence

$$
\left\{\begin{array}{l}
e_{1} \dot{e}_{1} \leq \frac{|a|}{2}\left(e_{1}^{2}+e_{2}^{2}\right)-a e_{1}^{2}+|a d| e_{1}^{2}+|a||c-d| e_{1}^{2}+l_{1} e_{1}^{2}-k_{1} e_{1}^{2} \\
e_{2} \dot{e}_{2} \leq \frac{1}{2}\left(e_{1}^{2}+e_{2}^{2}\right)-e_{2}^{2}+\frac{1}{2}\left(e_{2}^{2}+e_{3}^{2}\right)+l_{2} e_{2}^{2}-k_{2} e_{2}^{2} \\
e_{3} \dot{e}_{3} \leq \frac{|b|}{2}\left(e_{2}^{2}+e_{3}^{2}\right)+l_{3} e_{3}^{2}-k_{3} e_{3}^{2}
\end{array}\right.
$$

where 


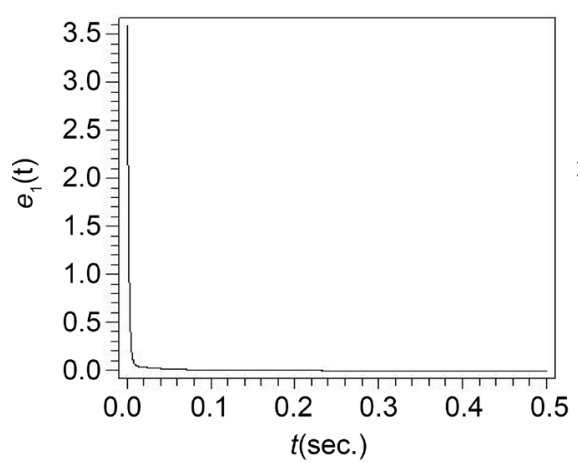

(a)

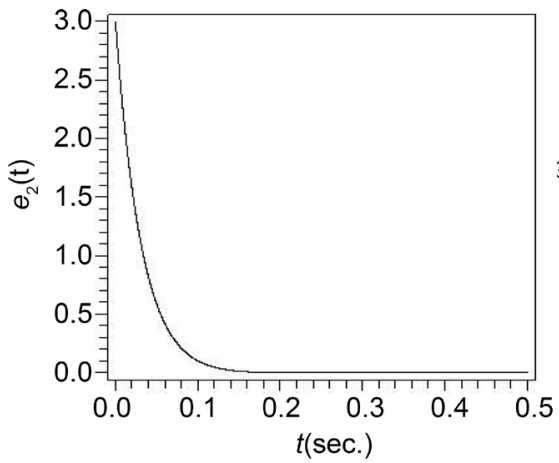

(b)

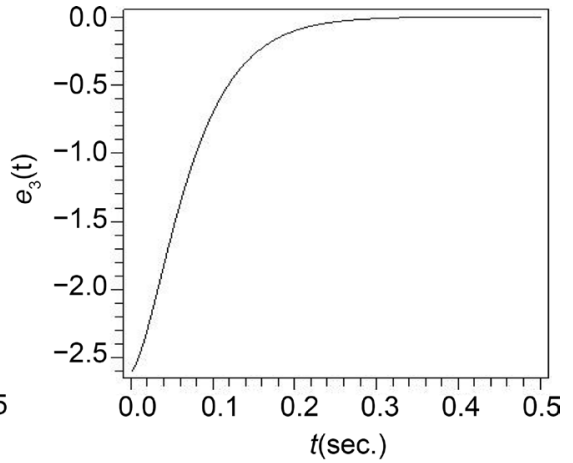

(c)

Figure 4. The history of the error (within $0.5 \mathrm{sec}$.).

$$
\left\{\begin{array}{l}
l_{1}=\frac{l_{f}+\left|\xi_{a}^{\prime}\right|\left(5+5|d|+5\left|\xi_{d}^{\prime}\right|+|c|+\left|\xi_{c}^{\prime}\right|\right)+\left|\xi_{a}\right|\left(5+5|d|+5\left|\xi_{d}\right|+|c|+\left|\xi_{c}\right|\right)+\left|d_{1}^{\prime}\right|+\left|d_{1}\right|}{\varepsilon} \\
l_{2}=\frac{\left|d_{2}^{\prime}\right|+\left|d_{1}\right|}{\varepsilon} \\
l_{3}=\frac{\left|\xi_{b}^{\prime}\right|+\left|\xi_{b}\right|+\left|d_{3}^{\prime}\right|+\left|d_{3}\right|}{\varepsilon} \\
\quad \text { and } l_{f}=|a|\left(5\left|\xi_{d}^{\prime}\right|+5\left|\xi_{d}\right|+\left|\xi_{c}^{\prime}\right|+\left|\xi_{c}\right|\right)
\end{array}\right.
$$

Choose Lyapunov function

$$
V(t)=\frac{1}{2}\left(e_{1}^{2}+e_{2}^{2}+e_{3}^{2}\right)
$$

We have

$$
\dot{V}(t)=e_{1} \dot{e}_{1}+e_{2} \dot{e}_{2}+e_{3} \dot{e}_{3}
$$

Substitute Equation (28) into Equation (31), obtain

$$
\begin{aligned}
\dot{V} & =\left(|a d|+|a||c-d|-\frac{a-1}{2}+l_{1}-k_{1}\right) e_{1}^{2} \\
& +\left(\frac{|a|+|b|}{2}+l_{2}-k_{2}\right) e_{1}^{2}+\left(\frac{1+|b|}{2}+l_{3}-k_{3}\right) e_{3}^{2}
\end{aligned}
$$

If

$$
\left\{\begin{array}{l}
k_{1}>|a d|+|a||c-d|-\frac{a-1}{2}+l_{1} \\
k_{2}>\frac{|a|+|b|}{2}+l_{2} \\
k_{3}>\frac{1+|b|}{2}+l_{3}
\end{array}\right.
$$

is satisfied, we will obtain $\dot{V}(t)<0$. According to Lyapunov stability theorem, the error system (24) will converge to zero when the error is not within the bound $\varepsilon$, i.e. system (21) and system (22) will achieve approximate synchronization.

Suppose the upper bounds of these disturbances and perturbation are 0.2, choose $\varepsilon=0.05$, substitute Equation (29) into Equation (32), after calculating 
we obtain if

$$
\left\{\begin{array}{l}
k_{1}>576 \\
k_{2}>21 \\
k_{3}>16
\end{array}\right.
$$

is satisfied, Equation (32) will be always true.

In the above simulation, let $\xi_{a}=0.2 \sin (t+3), \quad \xi_{b}=0.2 \cos (8 t+5)$, $\xi_{c}=0.2 \cos (3 t+5), \quad \xi_{d}=0.2 \sin (2 t), \quad \xi_{a}^{\prime}=0.2 \sin (t+1), \quad \xi_{b}^{\prime}=0.2 \cos (5 t+3)$, $\xi_{c}^{\prime}=0.2 \cos (5 t+1), \quad \xi_{d}^{\prime}=0.2 \sin (3 t+2), d_{1}, d_{2}, d_{3}, d_{1}^{\prime}, d_{2}^{\prime}, d_{3}^{\prime}$ are random from -0.2 to 0.2 . A time step of size 0.0001 (sec.) is employed and fourth-order RungeKutta method is used to solve Equation (21) and Equation (22). Let $k_{1}=580$, $k_{2}=30, k_{3}=20$, Figure 4 shows the history of $e_{1}(t), e_{2}(t), e_{3}(t)$ in the error system (24) within $0.5 \mathrm{sec}$. From Figure 4 , we can see that $e_{1}(t), e_{2}(t)$, $e_{3}(t)$ are steady near zero at last.

\section{Conclusion}

In this paper, a practical scheme is proposed for feedback synchronization with parameters perturbation and external disturbances. Lorenz system and the original Chua's circuit are used for detailed description. The simulation results show the feasibility of the method. According to Ref. [15], if all the feedback coefficients are larger than the largest Lyapunov exponent, two identical systems will be synchronized under ideal circumstance. In the paper, our scheme proved that high feedback coefficients will ensure more robust synchronization theoretically. The practical feedback should be bounded in a proper limit, so we have to control the error within a proper bound to obtain suitable feedback. The feedback will be smaller when the error is smaller. It's not hard for us to find a chance when the error between the drive system and the response system is small enough.

\section{Acknowledgements}

The work was supported by Natural Science Foundation of Liaoning Province (No. 201602034).

\section{References}

[1] Pecora, L.M. and Carroll, T.L. (1990) Synchronization of Chaotic Systems. Physical Review Letters, 64, 821-830. http://dx.doi.org/10.1103/PhysRevLett.64.821

[2] Carroll, T.L. and Pecora, L.M. (1991) Synchronizing Chaotic Circuits. IEEE Transactions on Circuits and Systems, 38, 453-456. http://dx.doi.org/10.1109/31.75404

[3] Wang, G.R., Yu, X.L. and Chen, S.G. (2001) Chaotic Control, Synchronization and Utilizing. National Defence Industry Press, Beijing.

[4] Wang, X.Y. (2003) Chaos in the Complex Nonlinearity System. Electronics Industry Press, Beijing.

[5] Chen, G.R. and Lü, J.H. (2003) Dynamical Analyses, Control and Synchronization of the Lorenz System Family. Science Press, Beijing.

[6] Murali, K. and Lakshmanan, M. (1996) Chaos in Nonlinear Oscillators Controlling 
and Synchronization. World Scientific, Singapore.

[7] Kapitaniak, T. (1996) Controlling Chaos: Theoretical and Practical Methods in Nonlinear Dynamics. Academic Press, London.

[8] Celka, P. (1996) Delay-Differential Equation versus 1D-Map: Application to Chaos Control. Physica D, 90, 235-241. http://dx.doi.org/10.1016/0167-2789(95)00243-X

[9] Agiza, H.N. and Yassen, M.T. (2000) Synchronization of Rössler and Chen Chaotic Dynamical Systems Using Active Control. Physics Letters A, 278, 191-197. http://dx.doi.org/10.1016/S0375-9601(00)00777-5

[10] Khadra, A., Liu, X.Z., et al. (2005) Impulsive Control and Synchronization of Spatiotemporal Chaos. Chaos, Solitons \& Fractals, 26, 615-636. http://dx.doi.org/10.1016/j.chaos.2004.01.020

[11] Guo, W.L., Chen, S.H., et al. (2009) A Simple Adaptive-Feedback Controller for Chaos Synchronization. Chaos, Solitons \& Fractals, 39, 316-321. http://dx.doi.org/10.1016/j.chaos.2007.01.096

[12] Agrawal, S.K., Srivastava, M., et al. (2012) Synchronization of Fractional Order Chaotic Systems Using Active Control Method. Chaos, Solitons \& Fractals, 45, 737 752. http://dx.doi.org/10.1016/j.chaos.2012.02.004

[13] Norelys, A.C., Manuel, A., et al. (2016) Adaptive Synchronization of Fractional Lorenz Systems Using a Reduced Number of Control Signals and Parameters. Chaos, Solitons \& Fractals, 87, 1-11. http://dx.doi.org/10.1016/j.chaos.2016.02.038

[14] Kajbaf, A., Akhaee, M.A., et al. (2016) Fast Synchronization of Non-Identical Chaotic Modulation-Based Secure Systems Using a Modified Sliding Mode Controller. Chaos, Solitons \& Fractals, 84, 49-57. http://dx.doi.org/10.1016/j.chaos.2015.12.002

[15] Wang, F.Q. and Liu, C.X. (2006) A New Criterion for Chaos and Hyperchaos Synchronization Using Linear Feedback Control. Physics Letters A, 360, 274-278. http://dx.doi.org/10.1016/j.physleta.2006.08.037

[16] Jiang, G.P. and Zheng, W.X. (2005) An LMI Criterion for Linear-State-Feedback Based Chaos Synchronization of a Class of Chaotic Systems. Chaos, Solitons \& Fractals, 26, 437-443. http://dx.doi.org/10.1016/j.chaos.2005.01.012

[17] Zhang, H. and Ma, X.K. (2004) Synchronization of Uncertain Chaotic Systems with Parameters Perturbation via Active Control. Chaos, Solitons \& Fractals, 21, 39-47. http://dx.doi.org/10.1016/j.chaos.2003.09.014

[18] Lorenz, E.N. (1963) Deterministic Nonperodic Flow. Journal of the Atmospheric Sciences, 20, 130-141. http://dx.doi.org/10.1175/1520-0469(1963)020<0130:DNF>2.0.CO;2

[19] Shil'nikov, L.P. (1994) Chua's Circuit: Rigorous Results and Future Problems. International Journal of Bifurcation \& Chaos, 4, 489-519. http://dx.doi.org/10.1142/S021812749400037X 
Submit or recommend next manuscript to SCIRP and we will provide best service for you:

Accepting pre-submission inquiries through Email, Facebook, LinkedIn, Twitter, etc. A wide selection of journals (inclusive of 9 subjects, more than 200 journals)

Providing 24-hour high-quality service

User-friendly online submission system

Fair and swift peer-review system

Efficient typesetting and proofreading procedure

Display of the result of downloads and visits, as well as the number of cited articles Maximum dissemination of your research work

Submit your manuscript at: http://papersubmission.scirp.org/

Or contactijmnta@scirp.org 